\title{
Development of Contractile Properties of Minced Muscle Regenerates in the Rat
}

\author{
Brtce M. Carlson ${ }^{1}$ and E. Gutmann \\ Department of Anatomy, Unizersity of Michigan, Ann Arbor, Michigan 48104: \\ and Institute of Physiology, Caechoslovak Academy of Sciences, \\ Prague 4, Krč, Czechoslovakia \\ Received March 30, 1972
}

\begin{abstract}
To test functional properties of regenerating skeletal muscle, the triceps surae muscles of 1-month-old rats were minced into $1-\mathrm{mm}^{3}$ pieces and replaced. Myogenic cells originating from the degenerating implanted muscle fraginents form a model of the original muscle and contractile activity is first detceted after 7-8 days. Early regenerates exhibited contractile properties of very slow muscles. Contraction time speeded up until by $30-40$ days it approached those of the normal fast muscles. The development of contractile function closely resembles that of normal muscles during the first postnatal weeks and it thus appears that the regenerating muscle undergoes a functional "developmental recapitulation." It is concluded that, despite a quantitative deficiency in the mass of regenerating muscle, those fibers which are present rapidly approach the normal condition in functional as well as morphological characteristics.
\end{abstract}

\section{Introduction}

The regeneration of mammalian skeletal muscle has been well documented for over a century, but the vast majority of work in the field of muscle regeneration has been morphologically oriented. Functional studics have been rare. Because many experimental models of muscle regeneration involve only local injury to a muscle, accurate recording of contractile properties of the regenerating tissue is difficult.

During the 1950's, Studitsky (14) designed an experimental model which is appropriate for studies on contractile properties of regenerating muscle. The technique involves completely removing a skeletal muscle, mincing it into small fragments and replacing the minced fragments into the bed of the removed muscle. From the implanted minced fragments myogenic cells arise which, together with the newly developing connective tissue, form a model of the muscle which was removed $(1,3,17)$. The regen-

1 Supported in part by grants from the Muscular Dystrophy Associations of America to both authors. Most of the experimental work was done while one of us (B.M.C.) was at the Institute of Physiology on an exchange visit sponsored by the U.S. and Czechoslovak Academies of Sciences. 
erated muscles, although considerably smaller than normal, possess good proximal and distal tendon connections. They became reinnervated ( 7 , $16)$, and contract after stimulation of their motor nerves $(7,14)$. Recently Salafsky (11) has recorded isometrically a number of contractile properties of mature minced muscle regenerates in normal and dystrophic mice. The present study is an analysis of certain contractile parameters of the regenerating minced gastrocnemius muscle in the rat. It was designed to determine when the regenerating muscle first acquires the ability to contract, the extent to which normal contractile properties are restored, and the course of functional maturation of regenerating muscle.

\section{Materials and Methods}

All animals used in these experiments were male Wistar rats. Experimental animals were 1 month old at the time of operation. Details of the operative procedure have been published $(1,3,15)$, but the essential features are given below. After anesthetization with sodium pentobarbital, the gastrocnemius, plantaris, and soleus muscles were entirely removed from one leg. Because the soleus is a slow muscle, it was discarded. The gastrocnemius and plantaris muscles are both fast, and contain a predominance of white and intermediate muscle fibers (13). These muscles were minced with scissors into $1-\mathrm{mm}^{3}$ fragments and were then implanted in place of the original muscles. At intervals from 7 to 100 days postoperatively animals were anesthetized with ether, the regenerates removed and weighed, and contractile properties determined. In the control series the gastrocnemius muscles were removed from 9- to 130-day-old, normal male animals.

For measurements of contractile behavior, a regenerated muscle was dissected free from its connective tissue adhesions with the surrounding tissues until the tendinous attachments at both the origin and insertion (Achilles tendon) were exposed. Ligatures were applied to both the proximal and distal insertion points, and the muscles were removed to a solution containing $149.8 \mathrm{~mm} \mathrm{Na}^{+}, 5.0 \mathrm{~mm} \mathrm{~K}, 2.0 \mathrm{~mm} \mathrm{Ca}^{++}, 148.0 \mathrm{~mm}$ $\mathrm{Cl}^{-}, 12.0 \mathrm{~mm} \mathrm{HCO}_{3}^{-}, 1.0 \mathrm{mM} \mathrm{H}_{2} \mathrm{PO}_{4}^{-}$, and $11.0 \mathrm{~mm}$ glucose at $p \mathrm{H} 7.2$ (8). The muscles were first equilibrated for $10 \mathrm{~min}$ in this oxygenated solution containing $0.01 \mathrm{~m}$ tubocurarine chloride (Merck) and then set up in the medium at $36 \mathrm{C}$ in a chamber containing Pt electrodes for massive direct stimulation (12). Resting tension for isometric recording was then determined, i.e., the initial length of muscle producing maximal twitch tension to direct stimulation, and recordings of contractile properties of twitch and tetanus were made by an automatic analyzer of muscle contraction properties (9). The twitch tension, the latency period (LP-stimulus artifact to first mechanical response), half (HCT) and full (FCT) contraction time (time to peak), half relaxation time (HRT-from peak to 
half amplitude of the spike), and the maximal rate of tension development (TCC) of the isometric twitch were recorded along with maximal tension output and maximal rate of tension development (TCC) of the isometric tetanus. Maximal rate of tension development is expressed as a time parameter ( $\mathrm{msec}$ ) defined as a time constant of contraction ( $\mathrm{TCC}$ ), i.e., the time necessary for reaching maximal force, assuming that contraction takes place from zero to maximum with maximal rate of tension development. In our experiments the half-time of this time constant was neasured. This TCC allows direct comparison of maximal rate of tension development between different animals without referring to other parameters as gram/cross-sectional area/second or gram/second/weight, etc. (10).

Maximal tetanic tension output was measured at optimal frequency (i.e., the frequency of stimulation giving the highest tension output; this generally corresponded to or was somewhat higher than the fusion frequency). A tetanus of 300 -msec duration was recorded, duration of the single stimulus being $1.0 \mathrm{msec}$.

After the determination of contractile behavior, many regenerates were fixed in Bouin's, serially cross-sectioned at $7 \mu$ and stained with hematoxylin and eosin or Heidenhain's aniline blue stain for connective tissue. In seven of the regenerates, total counts of muscle fibers were made in the cross sections containing the greatest area of muscle.

\section{Results}

Morphological Development. Gross and histological descriptions of regeneration of the minced gastrocnemius muscle have been published (1-3, 14,17 ), but the short summary below will serve as a morphological foundation upon which to base interpretations of the contractile properties of these regenerates. Within 2 or 3 days after the operation the first regenerative changes, in the form of myoblasts and early myotubes, are seen at the periphery of the implanted muscle mass. Throughout the next week regenerative activity increases greatly and spreads from the periphery of the implant toward the center. Meanwhile the minced mass has been molded into a shape resembling a muscle, and early connections have been established with the proximal and distal tendon stumps left behind at the time of the original operation. Early in the second postoperative week occasional cross striations are visible in new muscle fibers at the periphery of the regenerate, but most fibers are still in the myotube stage. By 10 days, all traces of the originally implanted muscle fibers have normally disappeared, and the regenerate consists entirely of immature muscle fibers and connective tissue. The succeeding weeks are marked by a maturation of the cellular and fibrous elements of the regenerate. Figure 1 illustrates a cross section of a typical mature minced muscie regenerate. 
Contractile Properties. The first contractile responses to direct stimulation were recorded in 7- to 8-day regenerates. The responses were just detectable on the oscilloscope, but the tensometer was not sufficiently sensitive for accurate recording. Reliable recordings could be made, however, in regenerates of 9 days and older. Contractile responses of early regenerates were extremely slow (Fig. 2). Recordings made 9 days after the operation (Table 1) showed that the contractile properties resembled closely those of normal muscles during the early postnatal period (Table 2 ). Throughout most of the regenerative process there is relatively little increase in the isometric twitch tension, but during the first month the LP, HCT, FCT, TCC, and HRT shorten rapidly (Table 1). By 30-40 days after the operation the HCT, FCT, and TCC have attained speeds which differ little from those of control muscles 48-60 days old. Although the LP and HRT of regenerates also speed up considerably during the first month of regeneration, they remain slower than the normal values by about $25 \%$ and $30-35 \%$ respectively. The LP, HCT, FCT, and TCC of regenerates

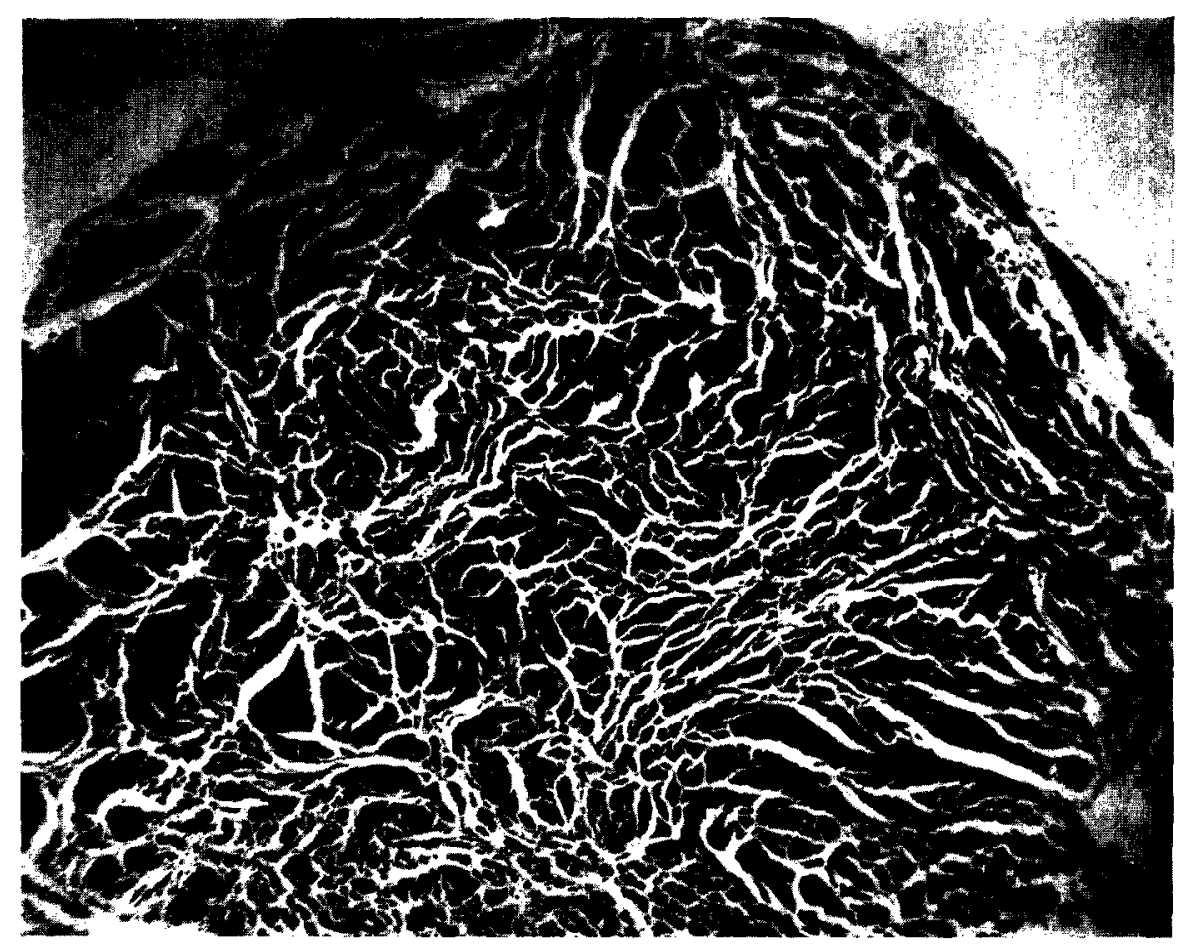

FIG. 1. Cross section through 73-day minced muscle regenerate in the rat (animal No. 7 of Table 3). The cross-sectional architecture of the regenerated muscle fibers is considerably less regular than normal. Hejdenhain's aniline blue stain. 


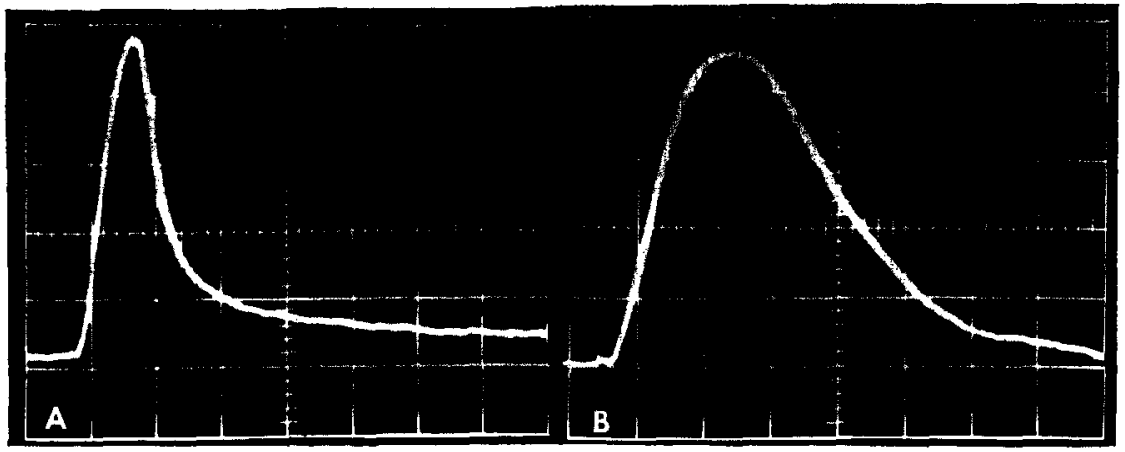

FIg. 2. A. Oscilloscope tracing of isometric twitch in the normal gastrocnemius muscle of 48-day-old rat. B. Oscilloscope tracing of isometric twitch in the contralateral gastrocnemius muscle which had been minced 18 days previously. Note the prolongation of both the contraction and relaxation phases of the twitch.

over 40 days old change relatively little in relation to those of normal muscles (Fig. 3), but HRT of regenerates continues to decrease up to 100 days.

In control muscles a very small prolongation of contraction and relaxation times can be observed in animals 60-130 days old. This corresponds to a small but continuous prolongation of contraction properties normally occurring with age (6), the prolongation being particularly marked in old age (5). As has been observed in other muscles of the rat $(4,6)$, the control muscle reaches its highest speed of contraction about 1.5-2 months after birth.

Fusion frequency increased from $30-40 / \mathrm{sec}$ in 12-day regenerates to

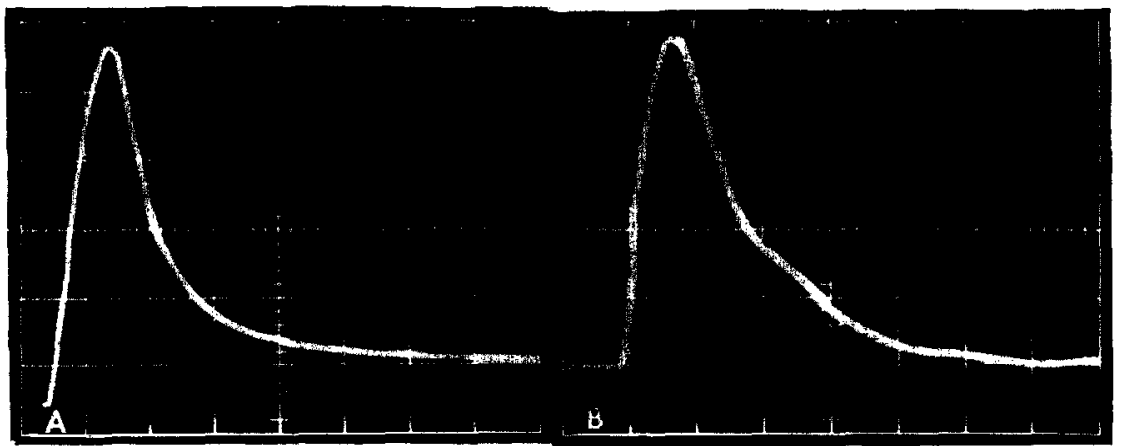

FiG. 3. A. Oscilloscope tracing of isometric twitch in the normal gastrocnemius muscle of a 105-day-old rat. B. Oscilloscope tracing of isometric twitch in the contralateral gastrocnemius muscle which had been minced 75 days previously. The contraction times are close to normal, but the relaxation phase is still prolonged. 


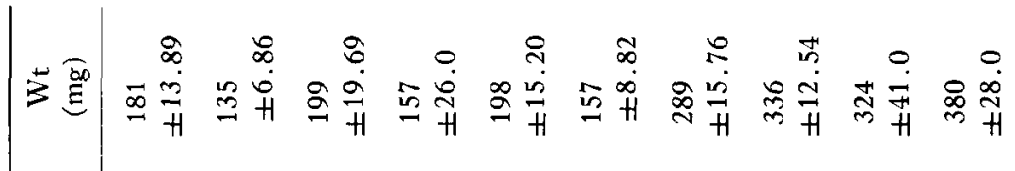

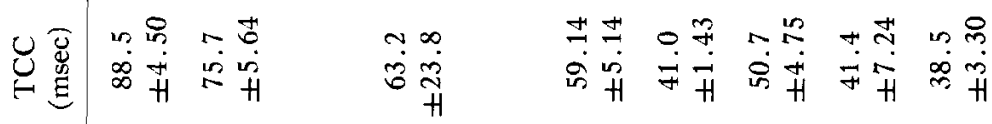

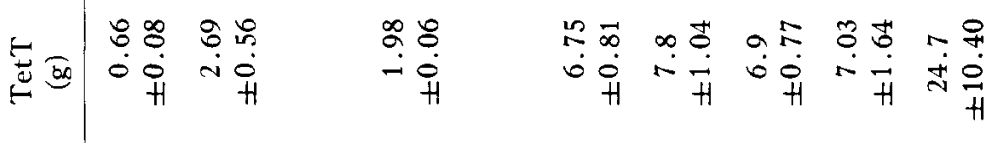

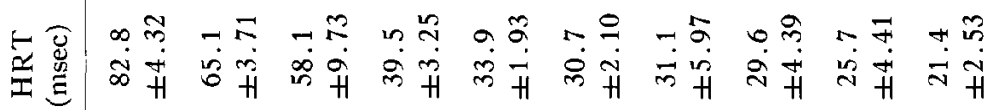

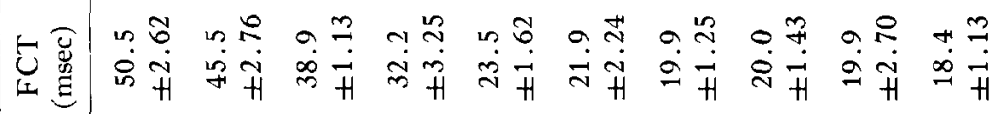

$$
\begin{aligned}
& \text { - }
\end{aligned}
$$

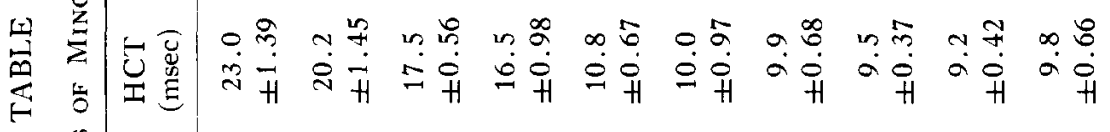

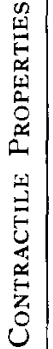

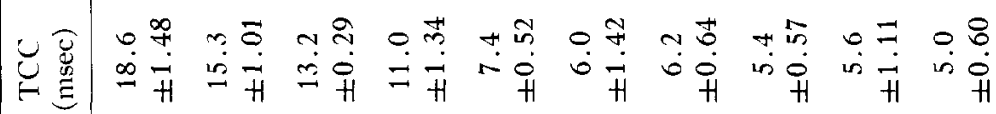

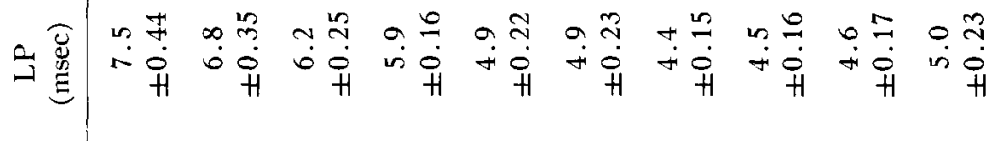

$$
\begin{aligned}
& \text { 岁 }
\end{aligned}
$$

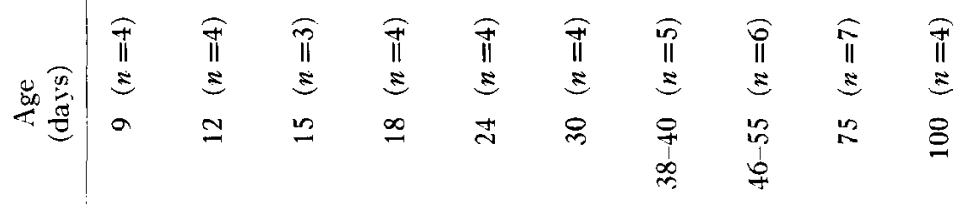




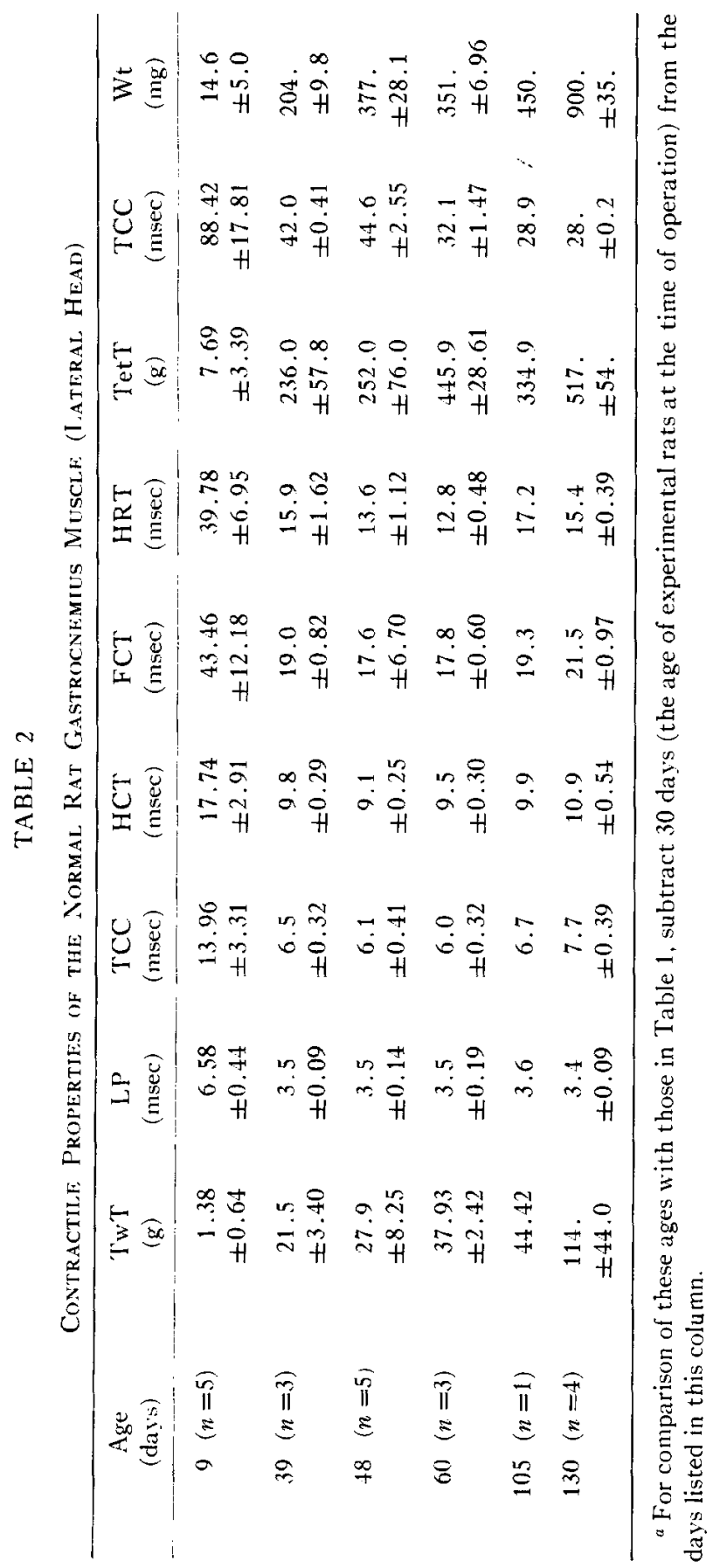


about $120 / \mathrm{sec} 30$ days after the operation and remained at the latter level in the older regenerates. Maximal tetanic tension output of regenerates increased considerably between 9 and 100 days after the operation (Table 1) but it remained far less than the maximal tetanic tension output of control muscles (the lateral head of the gastrocnemius). The TCC of the tetanus, although shortening progressively in regenerating muscle, did not attain normal values within 100 days. The pronounced difference in the TCC between twitch and tetanus in to a great extent explained by simultaneous activation of all muscle fibers in a twitch and the nonsimultaneous one during tetanus.

In seven mature regenerates, the number of nuscle fibers was counted in the region of the regenerate containing the largest area of muscle fibers. A comparison between number of muscle fibers per cross section and the tetanic tension produced by these aninlals in presented in Table 3 . There is a rough correlation between number of muscle fibers and tetanic tension, but in two cases (rat 2 and rat 6 ) there was considerable variation from the pattern of the other regenerates. There was no obvious histologic reason for these disparities.

In general the arrangement of muscle fibers in the cross sections examined was considerably less regular than normal (Fig. 1). There was also greater variation in the diameter of muscle fibers in regenerates than in normal muscle. These characteristics, along with a greater than normal amount of connective tissue, may account for the low tetanic tension observed in these regenerates.

\section{Discussion}

There is considerable correlation between the development of contractile function and morphological and histochemical fcatures of minced gastrocnemius muscle regenerates. Scattered bundles of immature muscle

TABLE 3

Comparison Between Number of Muscle Fibers of Regenerates and Tetanic Tension

\begin{tabular}{cccc}
$\begin{array}{c}\text { Animal } \\
\text { no. }\end{array}$ & $\begin{array}{c}\text { Age } \\
\text { (days) }\end{array}$ & $\begin{array}{c}\text { No. muscle fibers/ } \\
\text { cross section }\end{array}$ & $\begin{array}{c}\text { Tetanic tension } \\
(\mathrm{g})\end{array}$ \\
\hline 1 & 39 & 2100 & 4.50 \\
2 & 56 & 3105 & 9.97 \\
3 & 46 & 5063 & 6.70 \\
4 & 75 & 6869 & 6.72 \\
5 & 39 & 7452 & 9.50 \\
6 & 55 & 8540 & 4.74 \\
7 & 73 & 8810 & 9.08 \\
\hline
\end{tabular}


fibers containing cross-striations are first seen with the light microscope in 7- to 8 -day regenerates $(1,3)$, and it is at this time when the regenerating muscle is first noted to contract in response to direct stimulation. Nerve fibers are first demonstrable within the regenerate at the end of the first week or early in the second week (16), but motor end plates do not appear until considerably later. Using the Bielschowsky-Gros technique, Zhenevskaya (17) was able to demonstrate "motor end plate-like" structures in 3-week regenerates and more typical end plates by 4-5 weeks postoperatively. Although these results require substantiation with histochemical techniques, it would seem that the period during which the contraction and relaxation times change at the greatest rate is before or during the time when motor end plates appear to become fully established. It is also noteworthy that histochemical differentiation of different fiber types is first noticeable in regenerates early in the second month (13). This is the time when most contractile parameters have already returned to normal or near-normal values.

An examination of the functional parameters reveals a striking similarity between the contractile behavior of regenerating muscle and normally developing muscle. During the normal development of fast muscles in the rat a decrease of contraction times, half relaxation time, and an increase in the optimal frequency for repetitive stimulation occurs, the shortest contraction time being observed abut 30-40 days after birth (4). At birth both fast and slow muscles show approximately the same slow contraction times. Close (4) found the full contraction time of the newborn extensor digitorum longus (a very fast muscle) in the rat to be $56.5 \pm 5.3 \mathrm{msec}$. It dropped to $10.75 \pm 0.7 \mathrm{msec} 5$ weeks after birth and then increased slightly to adult values of $12.5 \pm 1.5 \mathrm{msec}$ at 100 days. These data during the first 1-2 months correspond to those of Gutmann and Melichna (6) who also observed a continuous, although small, prolongation of contraction time of the twitch during the following months. Since the gastrocnemius muscle, although essentially fast, is a mixed muscle with fast motor units predominating over the intermediate and slow motor units, its contraction time is slower than that of the extensor digitorum longus, but considerably more rapid than that of the slow soleus muscle (4).

When the regenerating gastrocnemius muscle has first developed the ability to contract (i.e., 9 days postoperatively) it is very slow indeed. The speed of contraction rapidly becomes faster until by $30-40$ days after the operation it has attained approximately maximal values. This roughly corresponds to the pattern of increasing speed of normal muscles after birth (6). Thus it appears that the regenerating muscle undergoes a process of "developmental recapitulation," at least with respect to the contractile characteristics of the twitch. The latency period decreases from the 
earliest regenerates until about 30 days, but remains somewhat longer than normal. This likely reflects irregularities in the development of the sarcotubular system, but confirmatory electron microscopic studies have not been performed.

The TCC of tetanus, reflecting the maximal rate of tension development, only slowly approaches control values. This could be due to differences in contractile characteristics of the regenerated muscle fibers (i.e., they might not be activated simultaneously during tetanus) or to the considerable increase in series elastic components resulting from the greater than normal amount of collagenous fibers in the muscle regenerates. The latter fact may also explain the relatively low tetanic tension output. The main reason for the low tetanic tension (as well as twitch tension) output is undoubtedly the relatively small mass of functional muscle fibers in minced muscle regenerates, which in the rat is always at least three or four times less than that of normal muscles. This is in marked contrast to the results of Salafsky (11), who found nearly complete functional return in minced gastrocnemius regenerates of the mouse. Because of a less vigorous conncctive tissue scarring response, minced muscle regeneration normally produces relatively larger muscles in mice than in rats. The extent and type of innervation of these regenerates is not well known with respect to functional categories.

In summary, the regenerating minced gastrocnemius muscle of the rat becomes functional early in the second postoperative week. Although these immature regenerates contract very slowly, the subsequent 3 weeks are marked by a rapid transition from slow to fast contractile properties so that by 30-40 days postoperatively most parameters tested approach those of a normal muscle of the same age. It is concluded that, despite a quantitative deficiency in the number of regenerating muscle fibers, those which are present rapidly approach the normal condition in functional as well as morphological characteristics. There is a striking parallel between the restoration of contractile properties of the regenerating gastrocnemius muscle and the development of contractile properties in the normal gastrocnemius during the first weeks after birth.

\section{References}

1. Carlson, B. M. 1968. Regeneration of the completely excised gastrocnemius muscle in the frog and rat from minced muscle fragments. J. Morphol. 125: $447-471$.

2. Carlson, B. M. 1970. Histological observation on the regeneration of mammalian and amphibian muscle, pp. 38-72. In "Regeneration of Striated Muscle and Myogenesis." $\Lambda$. Mauro, S. A. Shafiq, and A. T. Milhorat [Eds.]. Exerpta Medica, Amsterdam.

3. Carlson, B. M. In press. "The Regeneration of Minced Muscles." Karger, Basel. 
4. Close, R. 1964. Dynamic properties of fast and slow skeletal muscles of the rat during development. J. Physiol. 173 : 74-95.

5. Gutmann, E., V. Hanzlíkoví, and F. Vyskočll. 1971. Age changes in cross striated muscle of the rat. $J$. Physiol. 219 : 331-343.

6. Gutmann, E., and J. Melichna. 1972. Contractile properties of different skeletal muscles of the rat during development. Physiol. Bohemosloz. (in press).

7. Hsu, L. 1971. "The Role of Nerves in the Regeneration of Minced Muscle in Adult Anurans." Doctoral Dissertation. University of Michigan, Ann Arbor.

8. Liley, A. W. 1956. An investigation of spontaneous activity at the neuronuscular junction of the rat. J. Physiol. 132: 650-660.

9. RoHLÍČEK, V. 1968. An automatic analyzer of muscle contracttion. SNTL Tech. Dig. $61: 383-387$.

10. RohlíčEk, V., and E. GutMann. 1972. Time constant of contraction, a new expression of maximal rate of tension development. Physiol. Bohemoslor. (in press).

11. Salafsky, B. 1971. Functional studies of regenerated muscles from normal and dystrophic mice. Naturc (London) $229: 270-273$.

12. Sandow, A., and M. Brust. 1958. Contractility of dystrophic mouse muscle. Amer. J. Physiol. 194 : 557-561.

13. Snow, M. H. 1971. "Mctabolic Changes during Regencration of Minced Skeletal Muscle in the Rat." Doctoral Dissertation, University of Michigan, Ann Arbor.

14. Studitsky, A. N. 1959. "Experimental Surgery of Muscles." [in Russian]. Izdatel. Akad. Nauk SSSR, Moscow.

15. Studitsky, A. N., R. P. Zhenevskaya, and O. N. Rumyantseva. 1956. Fundamentals of the technique of restoration of muscles by means of the transplantation of minced muscle fragments. [in Russian]. Cesk. Morfol. 4: 331-340.

16. Zhenevskaya, R. P. 1954. Development of innervation of muscle, restored by means of the transplantation of minced muscle tissue. [in Russian]. Dokl. Akad. Nauk SSSR 96: 217-220.

17. ZhenevskayA, R. P. 1962. Experimental histologic investigation of striated muscle tissue. Rer'. Can. Biol. $21: 457-470$. 